\title{
Have we really understood when the efforts of laparoscopic liver resection are justified? - a complexity-based appraisal of the differential benefit
}

\author{
Francesca Ratti, Federica Cipriani, Guido Fiorentini, Marco Catena, Michele Paganelli, Luca Aldrighetti \\ Hepatobiliary Surgery Division, IRCCS San Raffaele Hospital, Milano, Italy \\ Contributions: (I) Conception and design: L Aldrighetti, F Ratti; (II) Administrative support: M Paganelli; (III) Provision of study materials or patients: \\ F Cipriani, G Fiorentini; (IV) Collection and assembly of data: M Catena, F Cipriani; (V) Data analysis and interpretation: F Ratti, L Aldrighetti; (VI) \\ Manuscript writing: All authors; (VII) Final approval of manuscript: All authors. \\ Correspondence to: Francesca Ratti, MD. Hepatobiliary Surgery Division, IRCCS San Raffaele Hospital, Via Olgettina 60, 20132 Milano, Italy. \\ Email: ratti.francesca@hsr.it.
}

\begin{abstract}
Background: The implementation of minimally invasive liver resection surgery (MILS) programs starts from procedures with a low degree of technical difficulty. Data regarding the real short-term advantage of laparoscopy according to technical difficulty are still lacking. The aim of the present study is to evaluate the differential benefit of laparoscopic over open technique according to the technical difficulty of the procedures and to investigate if efforts associated with laparoscopic approach are always justified.

Methods: Nine hundred and thirty-six MILS resections performed between 2005 and 2018 were stratified according to technical complexity (low, intermediate and high difficulty) and to approach (MILS or open) and matched in a 1:1 ratio using propensity scores to obtain three pairs of groups (Pair 1: Low-MILS and Low-Open, including 274 cases respectively; Pair 2: Int-MILS and Int-Open, including 237 patients respectively; Pair 3: High-MILS and High-Open, including 226 patients respectively).

Results: MILS approach resulted in a statistically significant lower blood loss, reduced morbidity, reduced and shorter time for functional recover and length of stay within all pairs. The evaluation of the differential benefit showed a greater advantage of laparoscopic approach in high degree procedures compared with intermediate and low degree, both in terms of blood loss $(-250$ and $-200 \mathrm{~mL}$ respectively) and morbidity rate $(-5.7 \%$ and $-4.1 \%$ respectively).

Conclusions: The favorable biological scenario associated with laparoscopic approach allows to obtain significant benefits in the setting of technically complex procedures. The commitment towards MILS approach should be therefore stronger in this setting, where the advantage of laparoscopy seems to be enhanced.
\end{abstract}

Keywords: Laparoscopic; liver; complexity; outcomes

Submitted Jul 01, 2020. Accepted for publication Aug 28, 2020.

doi: $10.21037 / \mathrm{hbsn}-20-562$

View this article at: http://dx.doi.org/10.21037/hbsn-20-562

\section{Introduction}

The feasibility of a wide range of laparoscopic procedures has been reported and documented, so that presently a large part of the portfolio of liver resections is reproduced by minimally-invasive approach (1-5). Due to the heterogeneous scenario of hepatic procedures, the need to focus on the concept of difficulty was perceived in the attempt to standardize it within objective parameters (6-9). In this perspective, several scores have been recently proposed, defining both technical complexity $(6,7,9)$ and the risk of intraoperative complications (8). These scores have been developed aiming to protect from the 
risk of discrepancy between the technical expertise of the surgeon and the degree of complexity of the procedure: consequently, difficulty scores have always been interpreted as a stairway "bottom-up".

Despite this, however, there is no evidence in the literature that the technical feasibility of laparoscopic liver resection is proportional to its clinical benefit. Indeed, paradoxically, recent data suggest that advantages of mini-invasiveness is more detectable in technically demanding proceduresi.e., resections in posterosuperior segments-compared to procedures characterized by a lower complexity-i.e., resections in anterolateral segments $(10,11)$.

Primary endpoint of the study is to analyze the degree of benefit (differential benefit) $(10,11)$ offered by laparoscopic compared to open surgery in different levels of technical complexity of the laparoscopic approach to define the best areas of its application in liver resections. Secondary endpoint was to confirm that advantages of minimally invasive techniques over the open counterpart are detectable within all levels of complexity and therefore justify, for liver resections, the efforts associated with this approach irrespectively of technical complexity. We present the following article in accordance with the STROBE reporting checklist (available at https://hbsn.amegroups.com/article/ view/10.21037/hbsn-20-562/rc).

\section{Methods}

\section{Study population and design}

The study was conducted in accordance with the Declaration of Helsinki (as revised in 2013). The study was approved by institutional ethics board (I Go MILS Registry) of San Raffaele Hospital, Milano, Italy and informed consent was taken from all individual participants. From January 2004 to May 2019, 2,971 liver resections were performed at the Hepatobiliary Surgery Division of San Raffaele Hospital in Milano. Intention-totreat approach was laparoscopic in 1,032 cases and open in 1,939 cases. Two levels of stratification of the overall series of liver resections were applied. The first stratification was performed according to technical difficulty and lead to the identification of three groups: the low, the intermediate and the high difficulty (see later for details regarding definition of complexity) (6). After this, a second stratification was performed according to approach (laparoscopic and open) to obtain six paired groups of patients [Pair 1: Lowminimally invasive liver resection surgery (MILS) and
Low-Open, including 274 cases respectively; Pair 2: IntMILS and Int-Open, including 237 patients respectively; Pair 3: High-MILS and High-Open, including 226 patients respectively]. Matching by propensity scores was performed between lap and open cases within the same level of difficulty in order to overcome possible inclusion bias. After that, the differential benefit for one technique over the other was calculated among groups. Details regarding the study design are provided in Figure 1.

Differential benefit was defined as the delta of advantage provided by the surgical approach (MILS versus open) among low, intermediate and high difficulty procedure $(9,10)$. The difference between laparoscopic and open resections was calculated as mean or median differences of values (for categorical and continuous variables respectively); the degree of advantage was compared between couples of cohorts (low and intermediate, low and high and intermediate and high): the higher difference was considered the final value of differential benefit. The differential benefit was calculated between the three cohorts both after excluding converted patients (pure differential benefit) and including them (risk-adjusted differential benefit). In order to calculate the differential benefit, intraoperative blood loss and postoperative 90-day morbidity were used as outcome indicators.

\section{Results validation by bootstrapping analysis}

To overcome possible bias of patients selection within groups, a non-parametric bootstrap process was applied on the original cohorts of low, intermediate and high difficulty resections as a resampling method in order to empirically estimate the sampling distribution. This method allowed to derive repeated samples (with replacement) from the original dataset thus mimicking the variability seen when taking samples from a population, and therefore was used to analyze the resulting distribution of the parameters of interest across the bootstrapped samples without relying on a particular distributional assumption.

\section{Preoperative workup}

Preoperative workup of all patients included liver function tests (to assess Child-Pugh classification) and serum tumor markers, abdominal ultrasonography, thoracoabdominal imaging (CT scan and/or MRI) and upper gastrointestinal (GI) endoscopy. Patients who were potential candidates for resection were systematically evaluated at weekly 


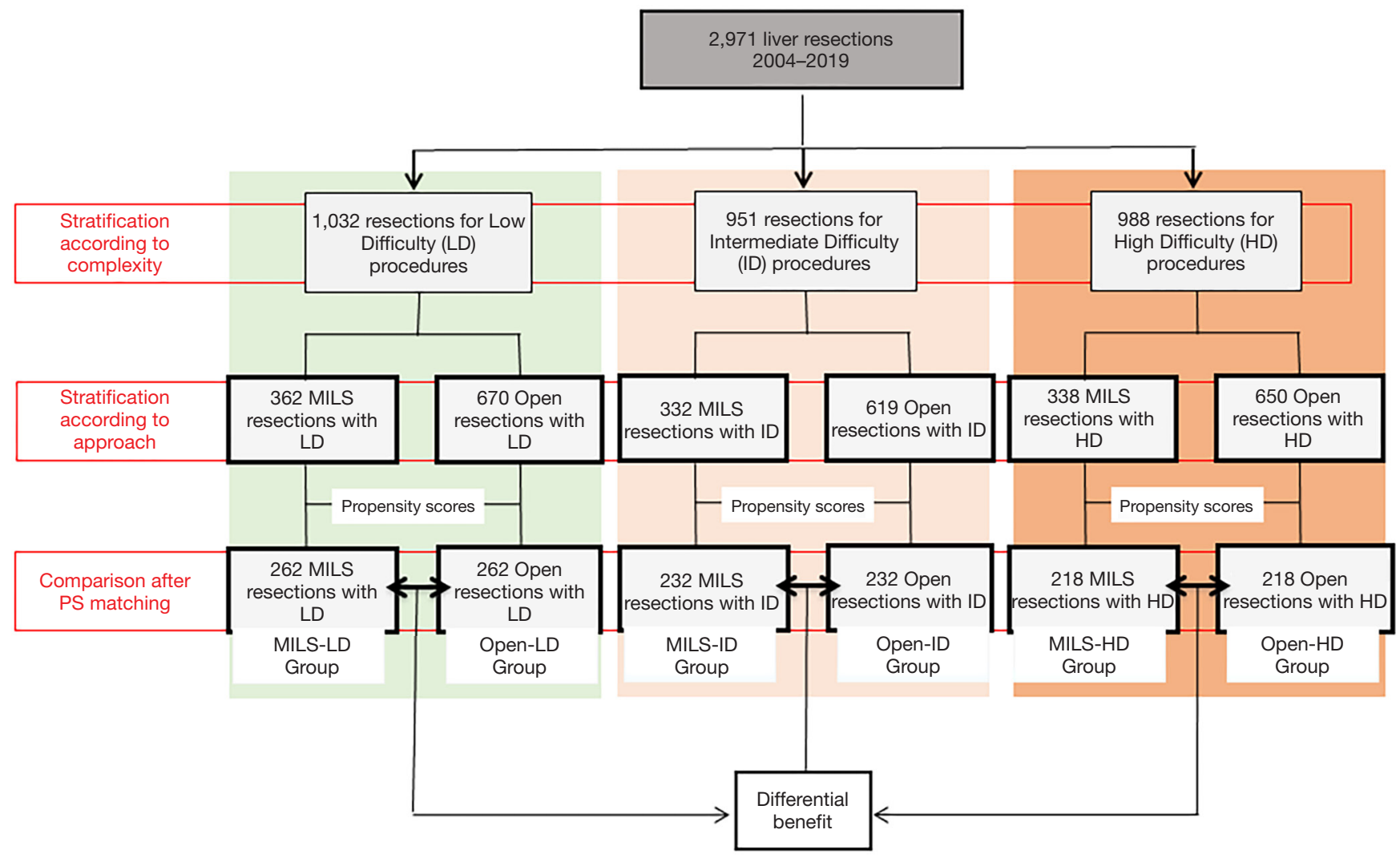

Figure 1 Study design. MILS, minimally invasive liver resection surgery. PS, propensity score.

multidisciplinary meetings, including liver surgeons, hepatologists, radiologists and medical oncologist to define the final indication for the surgical procedure and both the type and the resection technique.

\section{Definition of difficulty}

A preoperative index of difficulty for each operation was assessed using the Difficulty Score developed in the setting of laparoscopic resections by Ban-Wakabayashi (6), which is based on five preoperative factors (tumor location, extent of liver resection, tumor size, proximity to major vessels and liver function) and allows to define a final score by the sum of the five factors (6). Basing on the final score, each operation is then classified into three different categories: low [1-3], intermediate [4-6] and high difficulty [7-12].

\section{Surgical technique}

Laparotomy was performed through a right subcostal extended to midline incision. For laparoscopic resections the patient was placed in the "French" position, with the first surgeon standing between the patient's legs and one assistant on each side. Usually a 5 -trocar configuration was used with a $15-\mathrm{mm}$ port to house the $30^{\circ}$ laparoscope. Hepatic transection was performed by an alternating use of the SonoSurg system (Olympus, Tokyo, Japan), the bipolar forceps and an energy device (12). Pringle's maneuver was routinely used to control intraoperative bleeding (excluding patients requiring resections in the left lateral sector) both in laparoscopic and in open resections. Resection of three or more liver segments was considered a major hepatectomy (13).

\section{Perioperative management}

The enhanced recovery after surgery (ERAS) fast-track protocol was implemented into clinical practice for MILS and open resections in order to enhance the functional recovery. Characteristics of the institutional protocol are described elsewhere $(4,14)$. The patient is considered 
functionally recovered when all the following criteria are fulfilled: adequate pain control with oral analgesics; independent mobilization (mobile at preoperative level); tolerance of liquids and solid food; normal or decreasing serum bilirubin; no intravenous fluids; absence of complications.

\section{Outcome evaluation}

Data regarding general characteristics of patients and disease were recorded. Intraoperative and postoperative outcome were evaluated, including morbidity and mortality. Postoperative complications were reviewed for 90 days following liver resection and were graded retrospectively according to Dindo-Clavien classification of surgical complications (15). Postoperative mortality was defined as any death during postoperative hospitalization or within 90 days after resection. Postoperative liver failure was defined according to ISGLS definition. The pure and risk-adjusted differential benefit were calculated as described before for intraoperative blood loss and postoperative complications.

\section{Statistical analysis}

In order to overcome a possible allocation bias to open or laparoscopic approach, groups were matched using the propensity score with a small caliper (0.2) for the purpose of the present study. The following characteristics of patients and disease were used as covariates for matching: age, American Society of Anesthesiologists (ASA) score, gender, number of lesions, size of lesions, topography of lesion, type of resection. Bootstrapped groups were created from original cohorts in order to internally validate results. Demographic, pathologic, operative details, and surgical outcomes between groups were compared using the $\chi^{2}$ test or Fisher's exact test for categorical data and the MannWhitney $U$ test for ordinal data. All data were expressed as mean plus the standard deviation or median and range. Significance was defined as $\mathrm{P}<0.05$. All analyses were performed using the statistical package SPSS 18.0 (SPSS, Chicago, IL, USA).

\section{Results}

The study population consisted of 2,971 liver resections performed between 2004 and 2019, including 1,032 resections classified as low difficulty, 951 classified as intermediate difficulty and 988 classified as high difficulty procedures. After stratification of resections according to surgical technique and after application of the 1:1 matching process, three pairs of groups were obtained, with the following characteristics Pair 1: Low-MILS and Low-Open, including 274 cases respectively; Pair 2: Int-MILS and IntOpen, including 237 patients respectively; Pair 3: HighMILS and High-Open, including 226 patients respectively.

Characteristics of patients are reported in Table 1. Groups resulted well balanced between the approaches in terms of comorbidities, ASA score, features of the liver parenchyma, etiology of liver disease.

As reported in Table 2, the indication to liver resection was similar between matched groups and, in general, the most frequently indications were hepatocellular carcinoma (HCC) and colorectal metastases among malignant lesions which constituted the largest part of the series within all the levels of technical complexity. Distribution of the type of liver resection was similar between the approaches within all pairs: minor resections were the most frequently performed procedures in low and intermediate difficulty groups.

Among laparoscopic liver resection, the conversion rate was $8.8 \%(n=23)$ in Low-MILS group, $11.6 \%(n=42)$ in Int-MILS group and $17.4 \%(\mathrm{n}=38)$ in High-MILS group. bleeding and oncological concerns were the commonest reasons for conversion, with no significant differences among levels of complexity.

Table 3 reports postoperative outcome in the six groups of patients. Within all levels of complexity, a significant reduction of blood loss and transfusion requirement was recorded when minimally invasive approach was applied.

The benefit in terms of reduction of complication rate was significantly associated with laparoscopic approach in the Low-Open ( $8.8 \%$ vs. $13.4 \%, \mathrm{P}<0.05)$, Int-Open $(18.1 \%$ vs. $24.6 \%, \mathrm{P}<0.05)$ and High-Open group ( $28 \%$ vs. $37.6 \%$, $\mathrm{P}<0.05)$. The perioperative benefit of laparoscopy allowed a faster functional recovery and a faster discharge within all levels of technical complexity. Reflecting different degree of complexity, procedures showed significantly different blood loss, morbidity, rate of conversion and time for functional recovery among different pairs.

\section{Differential benefit}

Figure 2 reports the differential benefit in terms of blood loss. In particular, high difficulty procedures were associated with a greater advantage compared with low and intermediate difficulty procedures both when the pure differential benefit-i.e., excluding converted patient- 


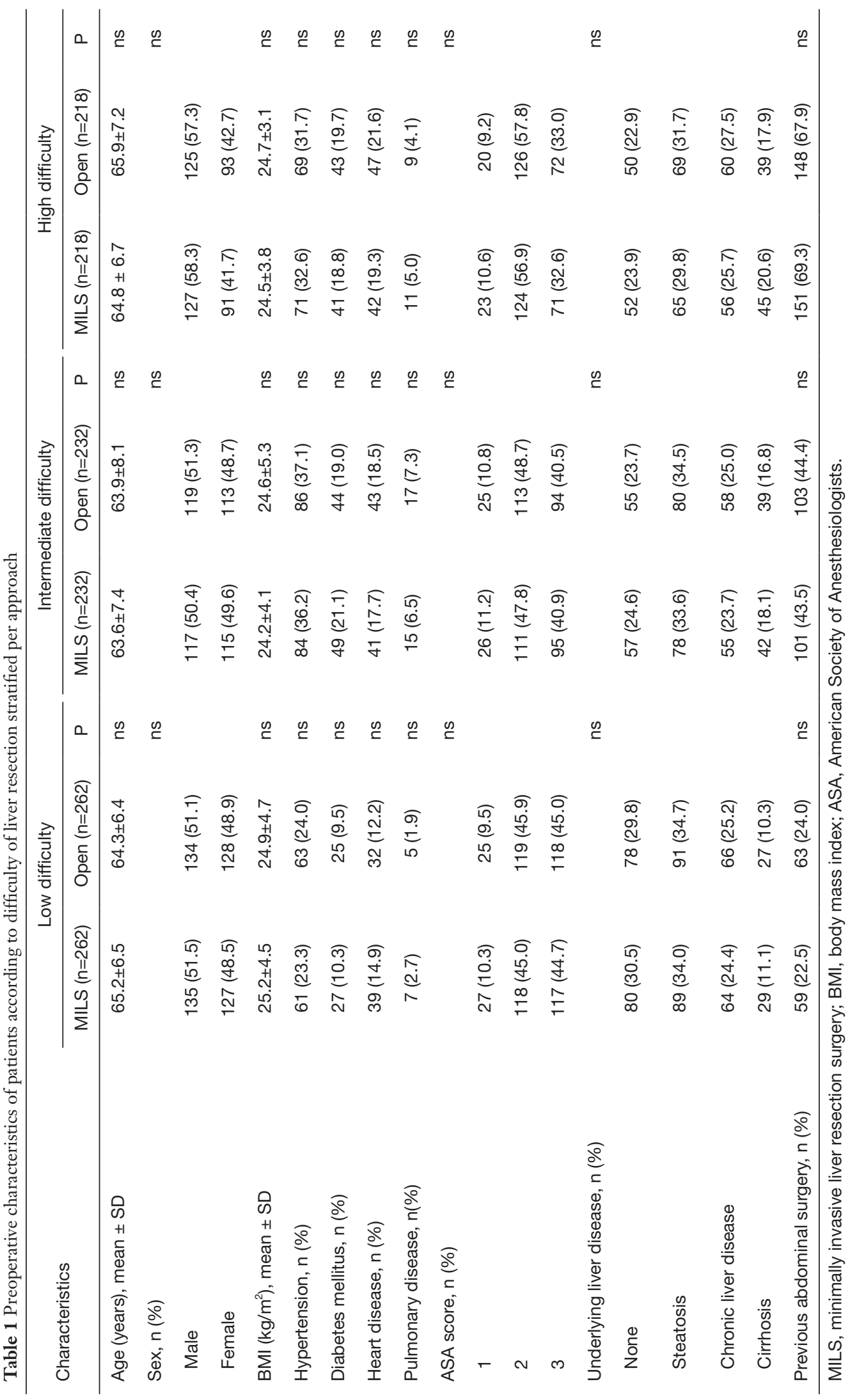




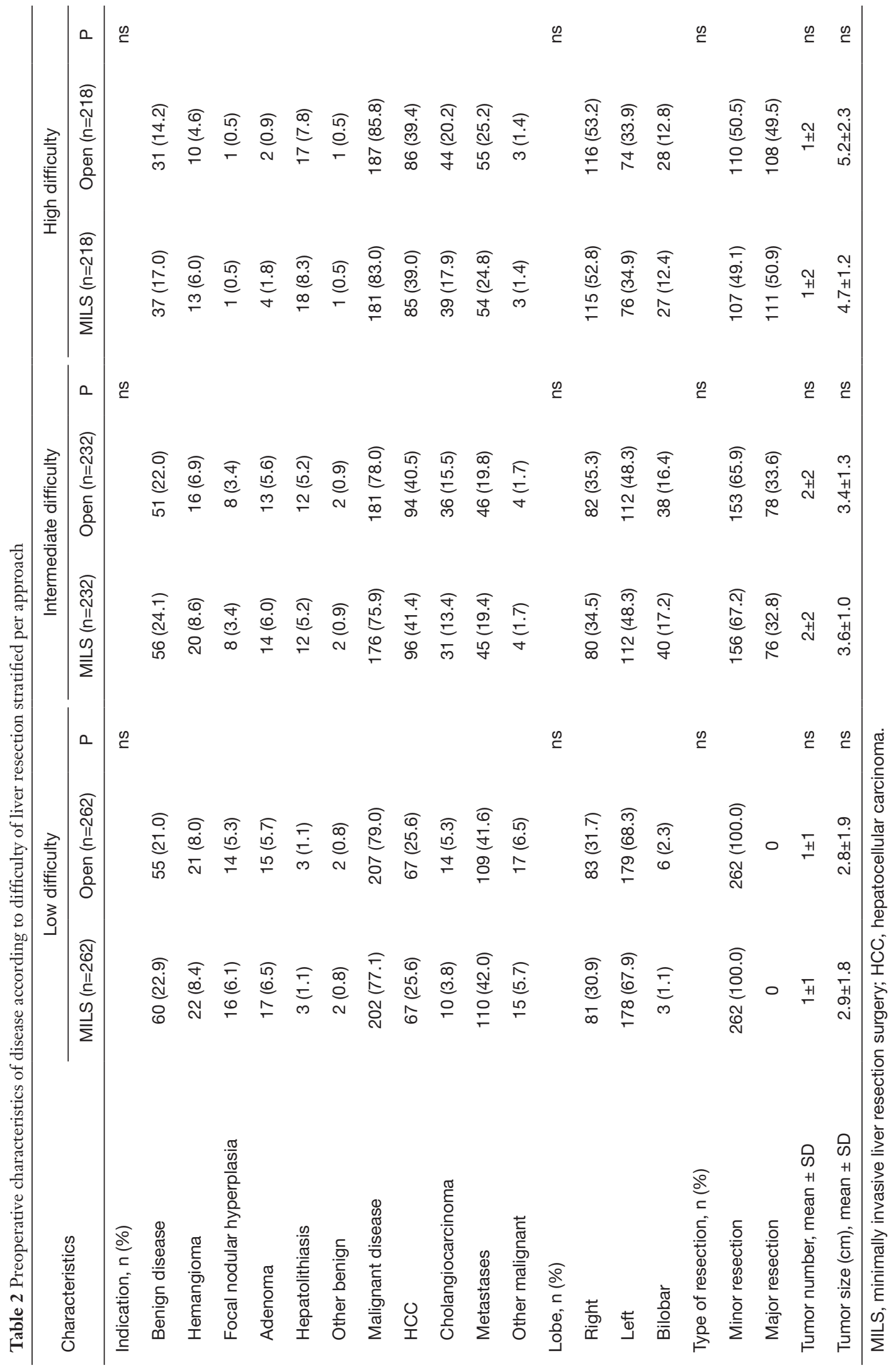




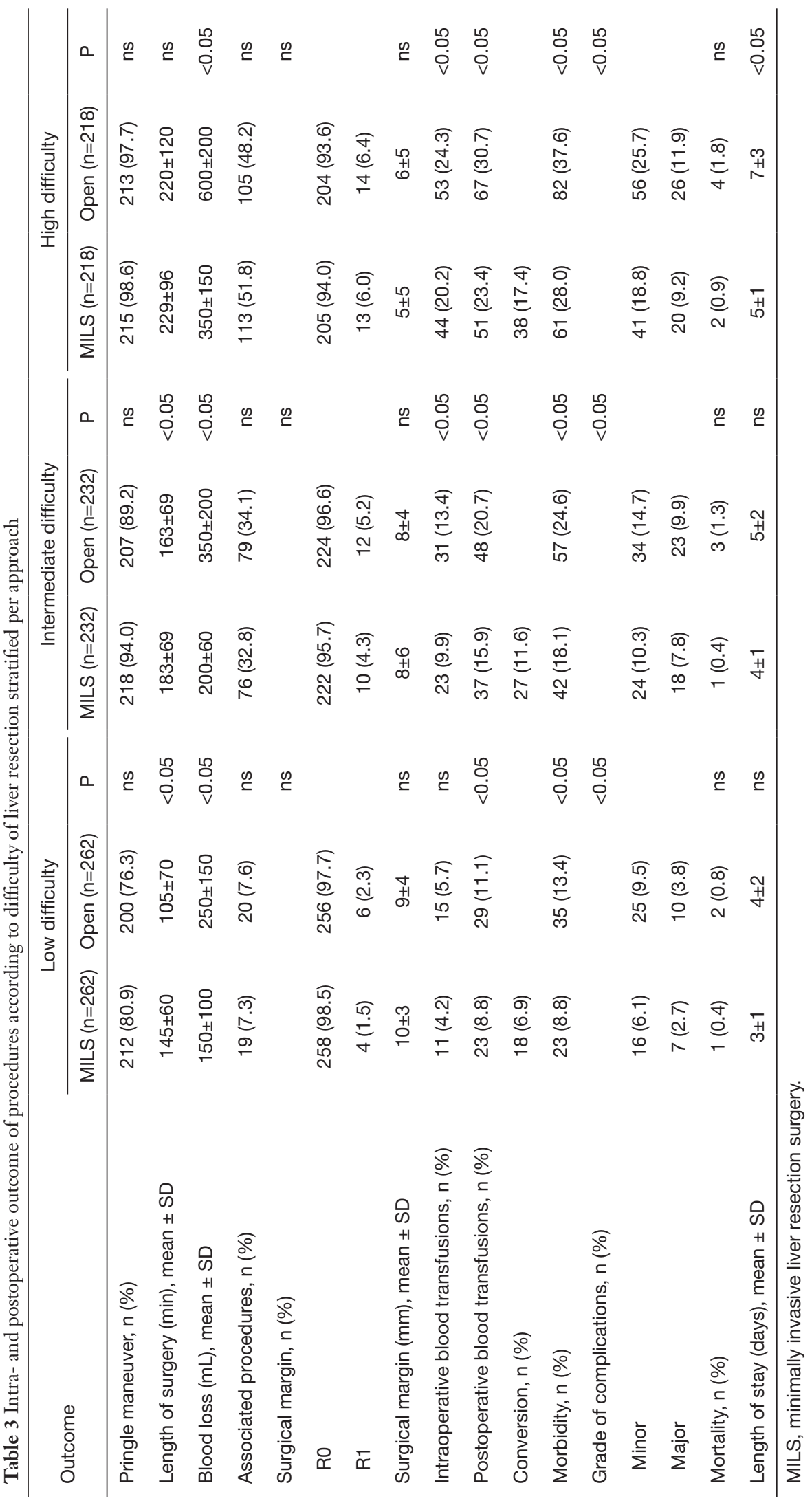




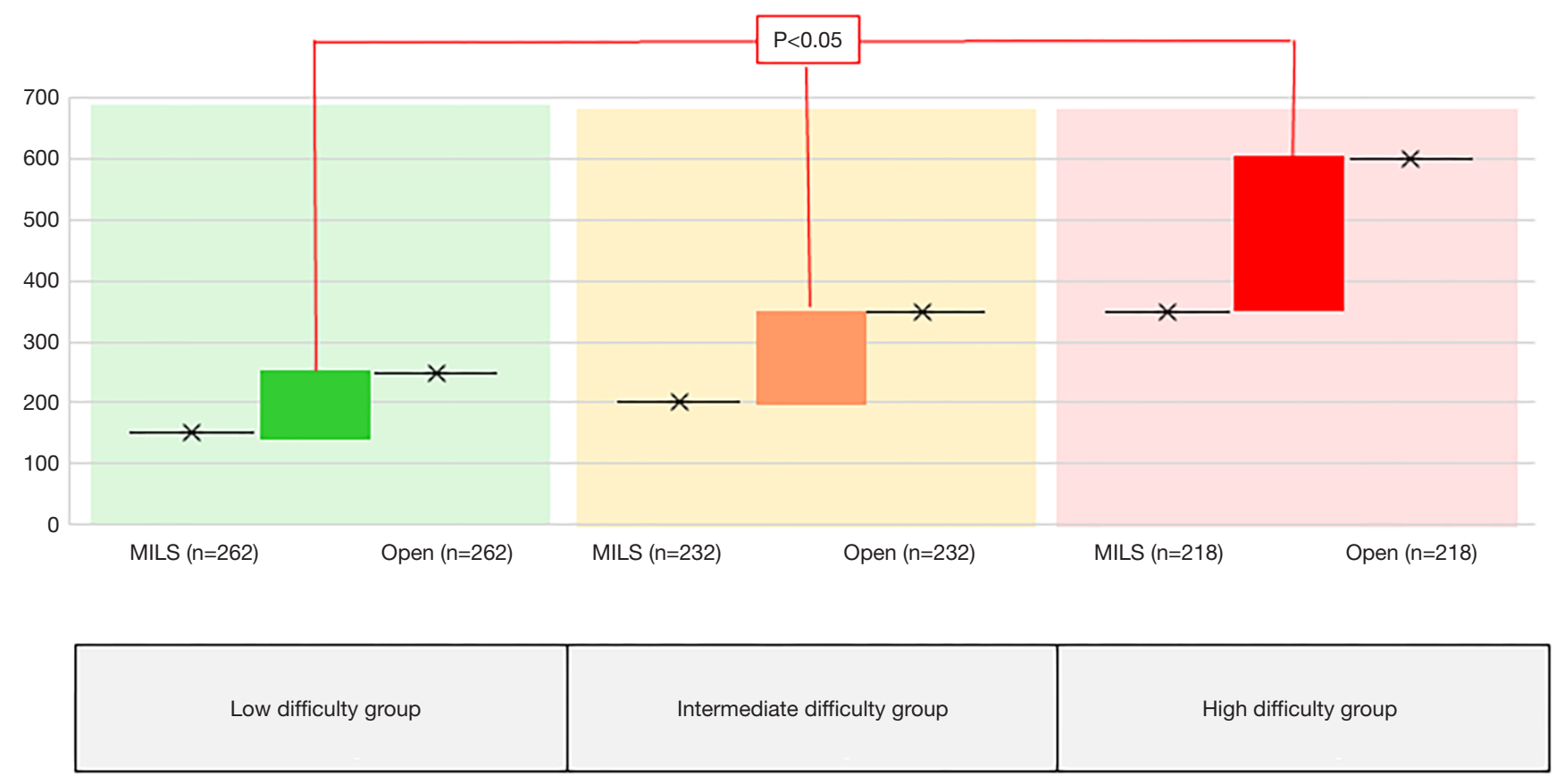

Figure 2 Pure differential benefit per blood loss. MILS, minimally invasive liver resection surgery.

(differential benefit: $150 \mathrm{~mL}, \mathrm{P}=0.032$ ) and the risk adjusted benefit-i.e., including conversions-(differential benefit: $110 \mathrm{~mL}, \mathrm{P}=0.041$ ) were calculated. Regarding morbidity rate, laparoscopic high difficulty procedures were associated with a greater degree of advantage compared with low and intermediate difficulty procedures both when the pure differential benefit (Figure 3)-(differential benefit: 7.7\%, $\mathrm{P}=0.018$ ) and the risk adjusted benefit (differential benefit: $5.9 \%, \mathrm{P}=0.026$ ) were evaluated.

The pure (without including conversions) and the risk-adjusted (including conversions) differential benefit were calculated both for blood loss and for morbidity: no significant differences between the two values was detected, ruling out the effect of conversion on determining the perioperative benefit of laparoscopic over open technique.

\section{Validation by bootstrapping analysis}

As a result of the bootstrapping process, 112 LowMILS were selected and compared with 112 Low-Open; similarly, 145 Int-MILS were selected and compared with 145 Int-Open and 160 High-MILS were selected and compared with 160 High-Open. The differential benefit in terms of blood loss was calculated in bootstrapped groups: a greater advantage in high difficulty compared with low and intermediate difficulty was documented both when the pure differential benefit (differential benefit: $180 \mathrm{~mL}$, $\mathrm{P}=0.033$ ) and the risk adjusted benefit (differential benefit: $130 \mathrm{~mL}, \mathrm{P}=0.046$ ) were calculated. Regarding morbidity rate, laparoscopic high difficulty resections were associated with a greater degree of advantage compared with low and intermediate difficulty when the pure differential benefit (differential benefit: 9.2\%, $\mathrm{P}=0.045$ ) and the risk adjusted benefit (differential benefit: $7.4 \%, \mathrm{P}=0.049$ ) were evaluated.

\section{Discussion}

The appraisal of the differential benefit stratified per complexity of MILS performed in the present study shows how the perioperative clinical advantage provided by the use of the laparoscopic approach is actually wider in procedures with a higher degree of technical complexity.

Scientific literature has so far targeted the need to demonstrate that the laparoscopic approach overall confers short term benefits over the open counterpart while maintaining an adequate standard of care in terms of 

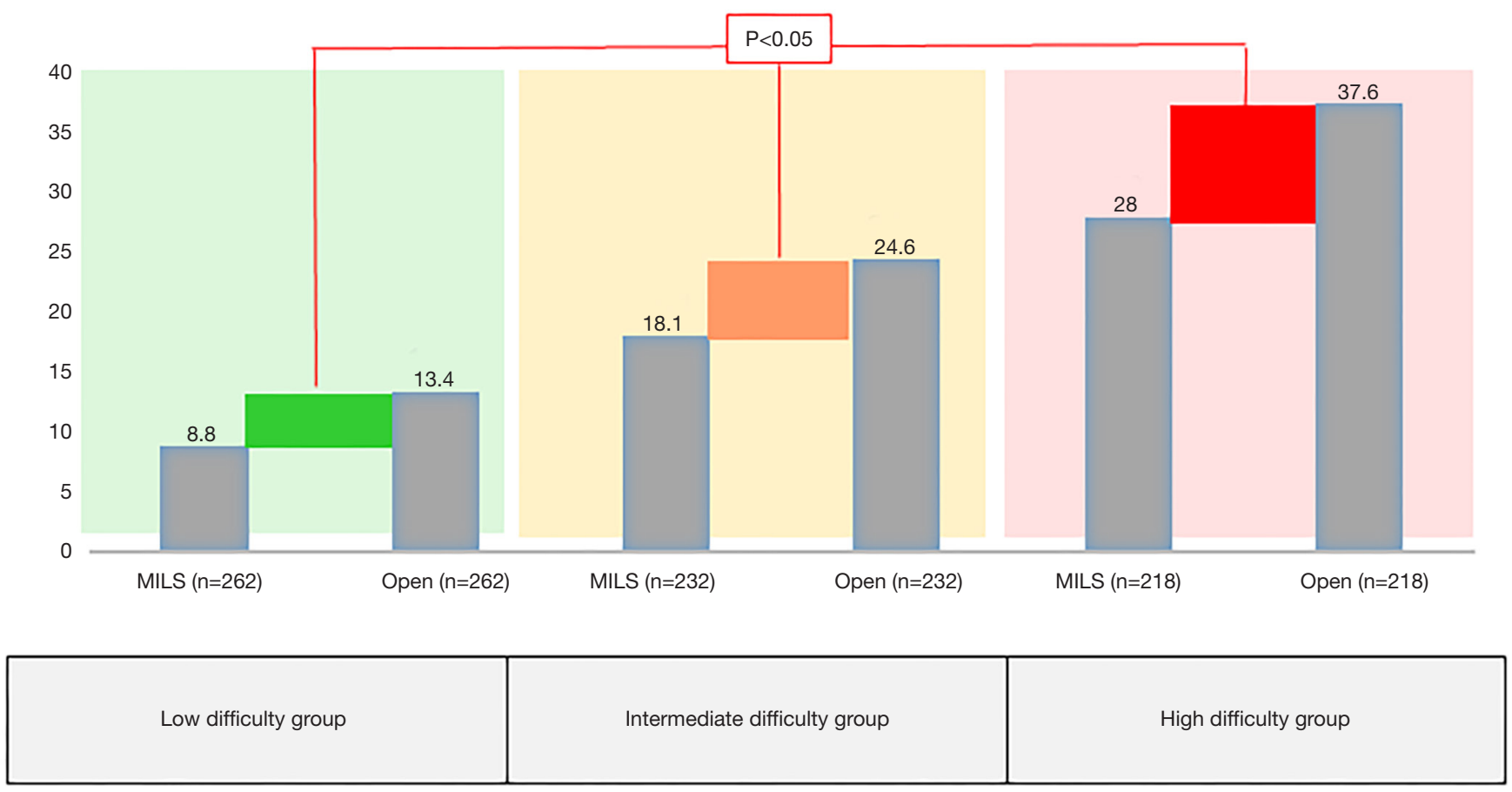

Figure 3 Pure differential benefit per morbidity. MILS, minimally invasive liver resection surgery.

oncological results (1-5). A large amount of literature has indeed been published during the last 15 years, comparing mixed series of laparoscopic and open liver resections or series selected by single or few items, from the technical (minor and major, type of resections, topography of lesions) $(1,3,16)$ or the pathological point of view (HCC, colorectal liver metastases, intrahepatic cholangiocarcinoma) (17-19). Even randomized controlled trials (RCTs) have been based only on type of resection or diagnosis $(20,21)$. Comparison of laparoscopic and open series selected by complexity defined by complexity scores has never been reported so far, as well as the degree of advantage of laparoscopic versus open approach in different profiles of complexity has never been analyzed.

Definition of difficulty scores have also been inspired by the need of guiding the stepwise learning curve in MILS: indeed, the learning curve of surgeons in MILS has also been studied in detail, to maintain a safety standard and to avoid the risk of a reduced performance because of the need of training $(22,23)$.

Recently, the concept of differential benefit was applied to the comparison between anterolateral and posterosuperior segments and a more pronounced advantage of laparoscopic resections was documented in the posterosuperior segments; anyway, no other specific insights about the area where the application of laparoscopy can offer best advantages in MILS are presently available (11). It is therefore now mandatory to evaluate the direction of MILS of the near future, to provide robust evidences to the surgical community and get prepared in the educational and allocation strategy perspective. In the present study, MILS approach is associated with clinical benefits over its open counterpart within all degrees of complexity but we aimed to understand in which setting of patients the advantage of laparoscopy is really justified and where instead its benefit is limited or even undetectable. The rationale was to understand if the efforts associated with laparoscopic approach (higher intraoperative costs, need for specific and dedicated training, possible longer operative times and therefore reduced number of procedures per day) are always worthwhile because of the clinical benefits irrespectively of complexity of the resection.

MILS approach resulted in a statistically significant lower blood loss, reduced morbidity, reduced and shorter time for functional recover and length of stay within all pairs. The evaluation of the differential benefit showed a greater advantage of laparoscopic approach in high degree procedures compared with intermediate and low degree, 
both in terms of blood loss ( -250 and $-200 \mathrm{~mL}$ respectively) and morbidity rate $(-5.7 \%$ and $-4.1 \%$ respectively). In other words, the higher is the complexity profile of the procedure by laparoscopic approach, the wider is the clinical advantage of being performed by MILS rather than open.

This assessment allows us to review the concept of difficulty score from a different and opposite perspective: indeed complexity is not only conceived a "bottom-up" stairway of progressive recruitment of cases depending on the step-by-step acquisition of surgical skills (complexity per training), but also as a "top-down" stratification of fields where mini-invasiveness is able to provide the greatest advantages (complexity per benefit). In this view, the degree of complexity should be addressed in an ascending scale during training in order to provide a good standard of safety during learning curve, but-after the learning curve has been completed - the use of complexity scores maintains its role, helping to define the best areas of clinical advantages provided by MILS in a descending scale of complexity. Complexity is therefore directly related both to training (i.e., lower training of surgeon-lower complexity of procedure; higher training of surgeon-higher complexity of procedure) and to clinical advantage (i.e., lower complexity of procedure-lower clinical advantage of MILS; higher complexity of procedure-higher clinical advantage of MILS), but-as a counterintuitive observation-MILS feasibility turns out to be inversely related to clinical advantage.

The reasons for the wider advantage of laparoscopy in complex resections have not yet been studied: evidences suggest that laparoscopy allows to provide a biological advantage to the patient (20).

The larger differential benefit may be hampered by the higher conversion rate and the outcome in converted cases, that can be worse than in cases completed by laparoscopy, especially when the conversion occurs because of intraoperative accidents: in the presence of a significant conversion rate indeed, the overall benefit could be jeopardized by severely unfavorable outcomes in converted patients, as already reported (24). In the present study adjusted differential benefit has been analyzed in addition to pure differential benefit to rule out specifically the impact of conversion.

In particular in the present study both the value of pure and adjusted differential benefit showed a wider advantage in patients with "high-complexity" compared to "intermediate-complexity" and "low-complexity" conferred by laparoscopic approach. this finding, together with the observation that the outcome indicators of the converted patients are worse than the respective cases completed laparoscopically but similar to the open cases, justifies the need to progressively extend the minimally invasive technique. Furthermore, it is even possible that the wider benefit of MILS in complex settings does not directly derive only from factors closely related to the laparoscopic technique, but also to the observation that in laparoscopy the strict application and adherence to fasttrack perioperative management protocols is easier and natural.

It can be speculated that, by increasing the technical complexity, in laparoscopy the advantage of the minimally invasive technique is maintained and enhanced by the ERAS protocol; vice versa, in complex open surgery both the advantage of mini-invasiveness and the systematic feasibility of the fast-track are, at least partially, lost (4). On the contrary, open resections with a low degree of technical complexity are better combined with the concept of fast track, leading the disadvantage given by laparotomy to be compensated by perioperative management.

Limitations of the present study are its retrospective nature and a possible underestimation or overestimation of surgical complexity in some procedures due to reported bias in difficulty scores (9).

Furthermore, the difficulty score was originally described by Ban et al. (6) specifically for MILS. Besides, it has still not been demonstrated that it can be implemented even in the setting of open liver resection. Consequently, a possible difference in grouping according to difficulty between laparoscopic and open approach cannot be excluded and constitutes a limitation in the present study, although the bias related to patient and tumor background was normalized via difficulty grouping, propensity score matching and bootstrapping.

Future prospective studies specifically designed to understand the correlation between technical complexity and advantage of laparoscopic approach in terms of homeostatic impairment and induction of surgical stress will be needed to precisely assess the biological background of the present findings.

In conclusion, laparoscopic approach allows to obtain significant benefits in the setting of technically complex procedures probably related to a favorable biological scenario, constituting the prerequisite for an adequate surgical outcome.

The commitment towards MILS approach should be therefore stronger in this setting, where-overcome the 
limit of technical challenges-the advantage of laparoscopy seems to be enhanced.

\section{Acknowledgments}

We acknowledge Dr. Kurt Carabott for the English revision of the manuscript.

Funding: None.

\section{Footnote}

Reporting Checklist: The authors have completed the STROBE reporting checklist. Available at https://hbsn. amegroups.com/article/view/10.21037/hbsn-20-562/rc

Data Sharing Statement: Available at https://hbsn. amegroups.com/article/view/10.21037/hbsn-20-562/dss

Conflicts of Interest: All authors have completed the ICMJE uniform disclosure form (available at https://hbsn. amegroups.com/article/view/10.21037/hbsn-20-562/ coif). LA serves as an unpaid editorial board member of Hepatobiliary Surgery and Nutrition. The other authors have no conflicts of interest to declare.

Ethical Statement: The authors are accountable for all aspects of the work in ensuring that questions related to the accuracy or integrity of any part of the work are appropriately investigated and resolved. The study was conducted in accordance with the Declaration of Helsinki (as revised in 2013). The study was approved by institutional ethics board (I Go MILS Registry) of San Raffaele Hospital, Milano, Italy and informed consent was taken from all individual participants.

Open Access Statement: This is an Open Access article distributed in accordance with the Creative Commons Attribution-NonCommercial-NoDerivs 4.0 International License (CC BY-NC-ND 4.0), which permits the noncommercial replication and distribution of the article with the strict proviso that no changes or edits are made and the original work is properly cited (including links to both the formal publication through the relevant DOI and the license). See: https://creativecommons.org/licenses/by-nc-nd/4.0/.

\section{References}

1. Abu Hilal M, Aldrighetti L, Dagher I, et al. The
Southampton Consensus Guidelines for laparoscopic liver surgery: from indication to implementation. Ann Surg 2018;268:11-8.

2. Morise Z, Aldrighetti L, Belli G, et al. Laparoscopic repeat liver resection for hepatocellular carcinoma: a multicentre propensity score-based study. Br J Surg 2020;107:889-95.

3. Cipriani F, Alzoubi M, Fuks D, et al. Pure laparoscopic versus open hemihepatectomy: a critical assessment and realistic expectations - a propensity score-based analysis of right and left hemihepatectomies from nine European tertiary referral centers. J Hepatobiliary Pancreat Sci 2020;27:3-15.

4. Ratti F, Cipriani F, Reineke R, et al. The clinical and biological impacts of the implementation of fast-track perioperative programs in complex liver resections: a propensity score-based analysis between the open and laparoscopic approaches. Surgery 2018;164:395-403.

5. Ciria R, Cherqui D, Geller DA, et al. Comparative shortterm benefits of laparoscopic liver resection: 9000 cases and climbing. Ann Surg 2016;263:761-77.

6. Ban D, Tanabe M, Ito $\mathrm{H}$, et al. A novel difficulty scoring system for laparoscopic liver resection. J Hepatobiliary Pancreat Sci 2014;21:745-53.

7. Kawaguchi Y, Fuks D, Kokudo N, et al. Difficulty of laparoscopic liver resection: proposal for a new classification. Ann Surg 2018;267:13-7.

8. Halls MC, Berardi G, Cipriani F, et al. Development and validation of a difficulty score to predict intraoperative complications during laparoscopic liver resection. $\mathrm{Br} \mathbf{J}$ Surg 2018;105:1182-91.

9. Halls MC, Cherqui D, Taylor MA, et al. Are the current difficulty scores for laparoscopic liver surgery telling the whole story? An international survey and recommendations for the future. HPB (Oxford) 2018;20:231-6.

10. Ratti F, Cipriani F, Fiorentini G, et al. Reappraisal of the advantages of laparoscopic liver resection for intermediate hepatocellular carcinoma within a stage migration perspective: Propensity score analysis of the differential benefit. J Hepatobiliary Pancreat Sci 2020;27:510-21.

11. Cipriani F, Ratti F, Paganelli $M$ et al. Laparoscopic or open approaches for posterosuperior and anterolateral liver resections? A propensity score based analysis of the degree of advantage. HPB (Oxford) 2019;21:1676-86.

12. Aldrighetti L, Pulitanò $\mathrm{C}$, Arru $\mathrm{M}$ et al. Ultrasonicmediated laparoscopic liver transection. Am J Surg 2008;195:270-2.

13. Terminology Committee of the International HepatoPancreato-Biliary Association. The Brisbane 2000 
terminology of liver anatomy and resections. HPB 2000;2:333-9.

14. Ratti F, Cipriani F, Reineke R, et al. Impact of ERAS approach and minimally-invasive techniques on outcome of patients undergoing liver surgery for hepatocellular carcinoma. Dig Liver Dis 2016;48:1243-8.

15. Dindo D, Demartines N, Clavien PA. Classification of surgical complications: a new proposal with evaluation in a cohort of 6336 patients and results of a survey. Ann Surg 2004;240:205-13.

16. Berardi G, Aghayan D, Fretland ÅA, et al. Multicentre analysis of the learning curve for laparoscopic liver resection of the posterosuperior segments. Br J Surg 2019;106:1512-22.

17. Ratti F, Fiorentini G, Cipriani F, et al. Laparoscopic vs open surgery for colorectal liver metastases. JAMA Surg 2018;153:1028-35.

18. Cipriani F, Fantini C, Ratti F, et al. Laparoscopic liver resections for hepatocellular carcinoma. Can we extend the surgical indication in cirrhotic patients? Surg Endosc 2018;32:617-26.

19. Ratti F, Fiorentini G, Cipriani F, et al. Perioperative and long-term outcomes of laparoscopic versus open lymphadenectomy for biliary tumors: a propensityscore-based, case-matched analysis. Ann Surg Oncol

Cite this article as: Ratti F, Cipriani F, Fiorentini G, Catena M, Paganelli M, Aldrighetti L. Have we really understood when the efforts of laparoscopic liver resection are justified?a complexity-based appraisal of the differential benefit. HepatoBiliary Surg Nutr 2022;11(3):363-374. doi: 10.21037/ hbsn-20-562
2019;26:564-75.

20. Fretland AA, Sokolov A, Postriganova N, et al. Inflammatory response after laparoscopic versus open resection of colorectal liver metastases: data from the Oslo-CoMet Trial. Medicine (Baltimore) 2015;94:e1786.

21. Wong-Lun-Hing EM, van Dam RM, van Breukelen GJ, et al. Randomized clinical trial of open versus laparoscopic left lateral hepatic sectionectomy within an enhanced recovery after surgery programme (ORANGE II study). Br J Surg 2017;104:525-35.

22. Ratti F, Barkhatov LI, Tomassini F, et al. Learning curve of self-taught laparoscopic liver surgeons in left lateral sectionectomy: results from an international multi-institutional analysis on 245 cases. Surg Endosc 2016;30:3618-29.

23. Aldrighetti L, Cipriani F, Fiorentini G, et al. A stepwise learning curve to define the standard for technical improvement in laparoscopic liver resections: complexitybased analysis in 1032 procedures. Updates Surg 2019;71:273-83.

24. Halls MC, Cipriani F, Berardi G, et al. Conversion for unfavorable intraoperative events results in significantly worse outcomes during laparoscopic liver resection: lessons learned from a multicenter review of 2861 cases. Ann Surg 2018;268:1051-7. 